\title{
A UNIFORM ASYMPTOTIC TURNING POINT THEORY FOR SECOND ORDER LINEAR ORDINARY DIFFERENTIAL EQUATIONS ${ }^{1}$
}

\author{
ERICH ZAUDERER
}

\begin{abstract}
The method of Cherry for obtaining uniform asymptotic solutions for a second order linear ordinary differential equation with a single turning point of first order is formally extended to the case where the equation has an arbitrary number of turning points of various orders. This follows a recent extension by Lynn and Keller of Langer's method to deal with the aforementioned more general problem.
\end{abstract}

1. Introduction. In a recent paper, Lynn and Keller [4] (hereafter referred to as L-K) developed a uniform asymptotic theory for homogeneous second order linear ordinary differential equations containing a large parameter and having an arbitrary number of turning points in some domain $D$. They represented the solutions of a given equation in terms of the solutions of a "related" or "comparison" equation, using a method introduced and developed by Langer [3] and employed by many others. In this method, the uniform asymptotic solution of the given second order equation is represented as a linear combination of a solution of the "related" equation and its derivative. The coefficients of these terms are specified as asymptotic expansions in inverse powers of the large parameter.

Cherry [1] developed a somewhat different method for obtaining a uniform asymptotic solution for the case of a single first order turning point for a second order differential equation. He also constructed a "related" equation, but he expressed the asymptotic solution of the given equation in terms of a solution of the "related" equation but not in terms of its derivative as well. The resulting uniform expansion takes a rather different form than that which arises from Langer's method. Its advantages over Langer's expansion are discussed in Cherry's paper [1] and are briefly

Received by the editors April 5, 1971.

AMS 1970 subject classifications. Primary 34E20.

Key words and phrases. Turning points, uniform asymptotic expansions, Cherry's method.

${ }^{1}$ This work was supported by the National Science Foundation under Grant No. GP-12835 and by the Department of Applied Mathematics, Weizmann Institute of Science, Rehovot, Israel.

(C) American Mathematical Society 1972 
touched on by Wasow [5]. Near the turning point, Cherry's result can be expanded out to give a result similar to Langer's.

In this paper we show that Cherry's method can be formally applied to the general problem considered by L-K. The calculations used in obtaining the coefficients in the uniform expansion, turn out to be more straightforward than those encountered by L-K in using Langer's method. Here a recursive system of first order ordinary differential equations must be solved while in L-K a recursive system of coupled first order equations arose. Further, we shall show that the solvability criteria which arise for both methods are essentially the same, and are in fact identical for a case we discuss in more detail. This is not unexpected since these conditions are given at the turning points where the Langer and Cherry expansions should be essentially equivalent.

With regard to systems of equations, which were also considered in L-K, it has been noted by Wasow [5] that Cherry's method does not seem to admit a straightforward generalization. This appears to be the case for higher order equations as well.

2. The uniform asymptotic solution. For the sake of brevity, we shall assume a familiarity with the contents of the paper by L-K as they apply to second order equations and refer to it when necessary. The given equation is assumed to have the form

$$
u^{\prime \prime}(Z)+\lambda^{2} R(Z, \lambda) u(Z)=0
$$

and we ask for an asymptotic solution of (1) as $|\lambda| \rightarrow \infty$ in some sector, for $Z$ restricted to some domain $D$. For large $|\lambda|$ we assume an asymptotic expansion for $R(Z, \lambda)$

$$
R(Z, \lambda) \sim \sum_{p=0}^{\infty} R_{p}(Z) \lambda^{-p}
$$

where the $R_{p}(Z)$ are either analytic or $C^{\infty}$ for all $p$, as assumed in L-K. A point $Z_{j}$ at which $R_{0}(Z)$ vanishes to order $m_{j}>0$ is said to be a turning point of order $m_{j}$. We assume there are $N+1$ turning points, i.e., $j=0,1, \cdots, N$. With $\mu$ equal to the sum of the orders of the turning points which lie in $D, \mathrm{~L}-\mathrm{K}$ introduce the related equation

$$
V^{\prime \prime}(\xi)+\lambda^{2} Q(\xi, \lambda) V(\xi) \equiv V^{\prime \prime}(\xi)+\left(\sum_{k=0}^{\mu} \gamma_{k} \xi^{k}\right) V(\xi)=0
$$

The $\gamma_{k}$ are functions of $\lambda$ and are to be specified. Following Langer's approach, L-K look for a solution of (1) in the form

(4) $u(Z, \lambda)=A(Z, \lambda) V\left[\lambda^{2 /(\mu+2)} \phi(Z)\right]+\lambda^{-\mu /(\mu+2)} B(Z, \lambda) V^{\prime}\left[\lambda^{2 /(\mu+2)} \phi(Z)\right]$ 
with the functions $A(Z, \lambda), B(Z, \lambda)$ and $\phi(Z)$ to be determined. They specify these functions, as well as the $\gamma_{k}$, in such a way that (4) is a formal nonsingular solution of (1). They also obtain uniform expansions which involve related equations having a form different from (3).

In terms of the related equation (3), the basic feature of Cherry's method is that we again ask for a solution of (1) in the form (4) but with $B(Z, \lambda) \equiv 0$. This implies, as we shall show, that $A(Z, \lambda)$ must have a specific functional form and that $\phi(Z)$ in (4) must be of the form $\phi(Z, \lambda)$, i.e., it must depend on $\lambda$ also. The problem is then reduced to determining the asymptotic expansion of $\phi(Z, \lambda)$ and specifying the $\gamma_{k}(\lambda)$ in (3). We apply this method first to a more general case where $Q(\xi, \lambda)$ in (3) is not specified a priori as a function of $\xi$ and then for the related equation given in (3). The latter case was discussed in detail in L-K.

Let a solution $u(Z, \lambda)$ of (1) have the form

$$
u(Z, \lambda)=A(Z, \lambda) V(\xi)
$$

where $V(\xi)$ satisfies the related equation (3) but with $Q(\xi, \lambda)$ as yet unspecified. Putting (5) into (1), with $\xi=\xi(Z, \lambda)$, gives

$$
\lambda^{2}\left(R-Q \xi^{\prime 2}\right) A V+A^{\prime \prime} V+\left(2 \xi^{\prime} A^{\prime}+\xi^{\prime \prime} A\right) V^{\prime}=0 .
$$

The coefficients of $V$ and $V^{\prime}$ must vanish separately. Thus

$$
2 \xi^{\prime} A^{\prime}+\xi^{\prime \prime} A=0 \text {. }
$$

Therefore, apart from a multiplicative constant,

$$
A=\left(\xi^{\prime}\right)^{-1 / 2} \text {. }
$$

On introducing this expression for $A$ into the coefficient of $V$ we obtain the equation

$$
\lambda^{2}\left[R-Q \xi^{\prime 2}\right]+\frac{3}{4}\left(\xi^{\prime \prime} / \xi^{\prime}\right)^{2}-\xi^{\prime \prime \prime} / 2 \xi^{\prime}=0 .
$$

To solve (9) we first expand $R$ and $Q$ asymptotically for large $\lambda$ and look for an asymptotic solution in the form

$$
\xi(Z, \lambda)=\sum_{n=0}^{\infty} \xi_{n}(Z) \lambda^{-n}
$$

Putting (10) into (9) leads to a recursive system of first order differential equations for the $\xi_{n}(Z)$, on equating coefficients of different powers of $\lambda$ to zero. Requiring the $\xi_{n}(Z)$ to be nonsingular, especially at the turning points $Z_{j}$, leads to conditions on the coefficients in the asymptotic expansion of $Q(\xi, \lambda)$. In view of $(8)$ we expect that $\xi^{\prime}(Z, \lambda) \neq 0$ at least for $\lambda=\infty$. Rather than show how $Q$ may be specified so that the $\xi_{n}(Z)$ are nonsingular, for the general case, we specialize our discussion to the case where $Q$ is given as in (3). This permits a more detailed comparison with 
the results in L-K. The formal analysis for this specific case is in fact more cumbersome than that for the case where an arbitrary $Q(\xi, \lambda)$ is considered.

Following L-K we expand $\gamma_{k}(\lambda)$ in (3) as follows

$$
\gamma_{k}(\lambda) \sim \lambda^{2(\mu-k) /(\mu+2)} \sum_{p=0}^{\infty} \gamma_{k p^{2}} \lambda^{-p}
$$

Asking for a solution of (1) in the form (4) with $B \equiv 0$, is obviously equivalent to replacing $\xi$ by $\lambda^{2 /(\mu+2)} \phi(Z, \lambda)$ in (5). $A(Z, \lambda)$ still has the same functional form (8) and putting (2) and (11) into (9) we have

$$
\sum_{p=0}^{\infty} \lambda^{2-p}\left[R_{p}(Z)-\sum_{k=0}^{\mu}\left(\phi^{\prime}\right)^{2} \phi^{k} \gamma_{k p}\right]+\frac{3}{4}\left(\phi^{\prime \prime} / \phi^{\prime}\right)^{2}-\phi^{\prime \prime \prime} / 2 \phi^{\prime}=0 .
$$

We look for a solution of (12) in the form

$$
\phi(Z, \lambda)=\sum_{n=0}^{\infty} \phi_{n}(Z) \lambda^{-n}
$$

Substituting (13) into (12), expanding out in powers of $\lambda$ and equating to zero the coefficients of different powers of $\lambda$ we obtain

$$
\begin{gathered}
\left(\phi_{0}^{\prime}\right)^{2} \sum_{k=0}^{\mu} \phi_{0}^{k} \gamma_{k 0}=R_{0}, \\
\phi_{1}^{\prime}+\left(\frac{R_{0}^{\prime}}{2 R_{0}}-\frac{\phi_{0}^{\prime \prime}}{\phi_{0}^{\prime}}\right) \phi_{1}=\frac{\phi_{0}^{\prime}}{2 R_{0}}\left[R_{1}-\sum_{k=0}^{\mu}\left(\phi_{0}^{\prime}\right)^{2} \phi_{0}^{k} \gamma_{k 1}\right], \\
\phi_{n}^{\prime}+\left(\frac{R_{0}^{\prime}}{2 R_{0}}-\frac{\phi_{0}^{\prime \prime}}{\phi_{0}^{\prime}}\right) \phi_{n}=\frac{\phi_{0}^{\prime}}{2 R_{0}}\left[\Phi_{n}\right] ; \quad n=2,3, \cdots,
\end{gathered}
$$

where the $\Phi_{n}$ contain terms involving $\phi_{n-r}$ and its derivatives for $0<r \leqq n$. The general solution of (16) is

$$
\phi_{n}(Z)=C_{n} \phi_{0}^{\prime}\left(R_{0}\right)^{-1 / 2}+\left(\frac{1}{2}\right) \phi_{0}^{\prime}\left(R_{0}\right)^{-1 / 2} \int_{Z_{0}}^{Z} \Phi_{n}(s)\left[R_{0}(s)\right]^{-1 / 2} d s
$$

where $Z_{0}$ is one of the turning points in $D$.

In discussing (14)-(17) we shall refer to equations appearing in L-K. Now, (14) is identical to (2.12) in L-K and the properties of its solution given there apply here as well. In particular, we have $\phi_{0}^{\prime}(Z) \neq 0$ in $D$ which is necessary in view of (8). Since the $\phi_{n}(Z)$ are required to be nonsingular in $D$ and $\left(R_{0}\right)^{-1 / 2}$ is singular at the turning points $Z=Z_{j}$, we must set the constants of integration $C_{n}$ in (17) equal to zero for all $n$. The particular solution in (17) also contains the singular factor $\left(R_{0}\right)^{-1 / 2}$. Thus the integral in (17) must be such that its product with $\left(R_{0}\right)^{-1 / 2}$ is nonsingular 
for all $Z=Z_{j}(j=0,1, \cdots, N)$. The necessary arguments depend on the order of the zeros of $R_{0}(Z)$ at the turning points and can be carried over directly from the discussion given in $\S 4$ of $\mathrm{L}-\mathrm{K}$. In particular, for $\phi_{1}(Z)$ we must have

$$
\begin{aligned}
& \int_{Z_{0}}^{Z_{j}}\left(R_{0}\right)^{-1 / 2}\left[R_{1}-\left(\phi_{0}^{\prime}\right)^{2} \sum_{k=0}^{\mu} \phi_{0}^{k} \gamma_{k 1}\right] d s=0, \\
& \frac{d^{q}}{d Z^{\alpha}}\left[R_{1}-\left(\phi_{0}^{\prime}\right) \sum_{k=0}^{\mu} \phi_{0}^{k} \gamma_{k 1}\right]=0 \text { at } Z=Z_{j}, \\
& q=0, \cdots, m_{j}-2, \quad j=0, \cdots, N .
\end{aligned}
$$

These equations are identical to equations (4.1)-(4.2) in L-K. For the functions $\phi_{n}(Z), n \geqq 2$, we get the conditions

$$
\begin{gathered}
\int_{Z_{0}}^{Z_{j}}\left(R_{0}\right)^{-1 / 2} \Phi_{n}(s) d s=0, \\
\frac{d^{q}}{d Z^{q}}\left[\Phi_{n}(Z)\right]=0 \quad \text { at } \quad Z=Z_{j}, \\
q=0, \cdots, m_{j}-2, \quad j=0, \cdots, N .
\end{gathered}
$$

It is readily seen that $\Phi_{n}(Z)$ has the form

$$
\Phi_{n}(Z)=R_{n}(Z)-\left(\phi_{0}^{\prime}\right)^{2} \sum_{k=0}^{\mu} \phi_{0}^{k} \gamma_{k n}+\cdots
$$

where the dots contain only terms $\gamma_{k r}$ for $r<n$. The possibility of satisfying the conditions (18)-(21) depends on being able to appropriately specify the $\gamma_{k n}, n=1,2, \cdots$, which appear in these equations. Using (22) in (20)-(21) we observe that the coefficient of the unspecified $\gamma_{k n}$ in the equations (18)-(21) [i.e., the $\gamma_{k n}$ which are to be solved for, for each value of $n$ ] are identical for all $n$. Interestingly enough, the coefficients of the $\gamma_{k n}$ which appear in the equations (4.1)-(4.2) and (4.4)-(4.5) in L-K are identical to ours. Thus, if their hypothesis (4.6), that the matrix of the coefficients of $\gamma_{k 1}$ in (18)-(19) has rank $\mu-1$, is satisfied we can solve for the $\gamma_{k n}$ at each step. We refer to L-K for a discussion of various ways in which the $\gamma_{k n}(n=1,2, \cdots)$ may be chosen.

In any case, the related equation (3) can be completely specified and a nonsingular formal solution of $(1)$ is obtained since the $\phi_{n}(Z)$ and consequently $\phi(Z, \lambda)$, are given once the $\gamma_{k n}$ are known.

3. Conclusion. We have shown that a uniform asymptotic solution of (1) can be obtained in the form

$$
u(Z, \lambda)=\left[\phi^{\prime}(Z, \lambda)\right]^{-1 / 2} V\left[\lambda^{2 /(\mu+2)} \phi(Z, \lambda)\right]
$$


where $V$ satisfies the related equation (3) and $\phi(Z, \lambda)$ is given by the asymptotic expansion (13). The expansion (23) is valid near the turning points $Z=Z_{j}$ of (1) and away from them. If we truncate the expansion (13) at $n=m$, we obtain an approximate solution of (1) with an error of the order $\lambda^{-m}$. We have seen that the algorithm for obtaining the terms $\phi_{n}(Z)$ in (13) leads to simple first order equations. This is in contrast to the coupled system of first order equations which arose in L-K. The matrix of the coefficients of the $\gamma_{k n}$ was shown above to be identical to that which occurs in L-K. We have not shown that the full equations for the $\gamma_{k n}$ are identical in general no matter whether Cherry's or Langer's method is used. (This is the case, however, for $\gamma_{k 0}$ and $\gamma_{k 1}$.) While this seems reasonable, a direct proof of this appears to involve a considerable effort. Now, L-K discuss other forms of the related equation and consider some specific turning point problems in detail. We note that Cherry's method can also be applied for more general related equations as was indicated above. Thus, we can write down expansions in terms of comparison equations other than (3), of the types L-K considered.

Finally, we observe that in Cherry's original paper [1] he used a different method for constructing his uniform asymptotic solution. Our method, which is more direct, seems to be suggested in a discussion of Cherry's method contained in Erdélyi's book [2].

\section{REFERENCES}

1. T. M. Cherry, Uniform asymptotic formulae for functions with transition points, Trans. Amer. Math. Soc. 68 (1950), 224-257. MR 11, 596.

2. A. Erdélyi, Asymptotic expansions, Dover, New York, 1956. MR 17, 1202.

3. R. E. Langer, The asymptotic solutions of ordinary linear differential equations of the second order, with special reference to a turning point, Trans. Amer. Math. Soc. 67 (1949), 461-490. MR 11, 438.

4. R. Y. S. Lynn and J. B. Keller, Uniform asymptotic solutions of second order linear ordinary differential equations with turning points, Comm. Pure Appl. Math. 23 (1970), 379-408. MR 41 \#5719.

5. W. Wasow, Asymptotic expansions for ordinary differential equations, Pure and Appl. Math., vol. 14, Wiley, New York, 1965. MR 34 \#3041.

Department of Mathematics, Polytechnic Institute of Brooklyn, Brooklyn, NEW YORK 11201 